\title{
A Stiff Person Case Admitted Phsysical Medicine and Rehabilitation Outpatient Clinic with Low Back Pain
}

\author{
Belgin KARAOĞLAN, Şeyda ÖTE, Ebru KÖSEOĞLU, Elif BALEVI \\ Department of Physical Medicine and Rehabilitation, Gazi University Faculty of Medicine, Ankara, Turkey
}

\begin{abstract}
Stiff-Person Syndrome (SPS) is a rare disorder that is characterized by stiffness and rigidity of the axial, abdominal, and extremity muscles. It is associated with several autoimmune diseases or paraneoplastic conditions. We report a case of SPS with Hashimoto's thyroiditis who complained of low back pain. Her physical examination was remarkable in that her abdominal and axial muscles were firm to palpation, and she had hyperlordosis of the spine. Active range of motion of the spine was limited, and she had a robotic gait. Electromyography demonstrated continuous motor unit activity in the agonist and antagonist muscles. Diagnosis of SPS was confirmed because antiglutamic acid decarboxylase was present in the cerebrospinal fluid. She favorably responded to intravenous administration of immunoglobulin, diazepam, and baclofen and stretching, strengthening, and range of motion exercises. Keywords: Back pain, Hyperlordosis, Stiff-Person Syndrome
\end{abstract}

\section{Introduction}

Stiff person syndrome (SPS) is a rare neurological disease that was first described by Moercsh and Woltman in 1956 as "stiff man syndrome" in 14 patients with complaints of stiffness in the dorsal, abdominal, and femoral muscles $(1,2)$.

Stiff person syndrome is an autoimmune disease that is characterized by the rigidity of the abdominal, axial, and extremity muscles; lumbar hyperlordosis; difficulty in moving; gait disturbance; and painful muscle spasms (1). Showing a fluctuating or stable course, it leads to disability (3). It has been reported that $65 \%$ of patients are dependent for their daily activities (4). The severity of the symptoms varies from mild to severe, and if the thoracic paraspinal and respiratory muscles are affected, it can be life threatening because of respiratory distress (5).

Apart from the aforementioned symptoms, the diagnosis of SPS is clinically established by the exclusion of other neurological diseases that cause stiffness and rigidity and by the absence of extrapyramidal and pyramidal symptoms (3). In electromyography (EMG), the presence of simultaneous and continuous motor unit activity in agonist and antagonist muscles and high titer of anti-glutamic acid decarboxylase (GAD) support the diagnosis (4). GAD antibodies are detected in $60 \%-80 \%$ of patients $(6,7)$.

Stiff person syndrome has been associated with other autoimmune diseases. Among 60 patients who were monitored by Dalakas et al. (6), it was most frequently observed in patients with type 1 diabetes mellitus (DM) in addition to pernicious anemia, vitiligo, and thyroiditis SPS. Five percent of patients have been reported to have the paraneoplastic condition $(1,3,6,8)$. In some cases that develop with neoplasms, such as breast cancer, anti-amphiphiles or anti-gephyrin autoantibody positivity has been reported $(9,10)$.

To treat the disease, symptomatic therapy and immunotherapy are essential. In symptomatic therapy, physical therapy, 
exercises, benzodiazepines, baclofen, tiagabine, and levetiracetam are used; in immunotherapy, particularly intravenous immunoglobulin (IVIG), plasmapheresis, rituximab (anti-CD20), and corticosteroids are used $(8,11)$.

\section{Case Report}

A 26-year-old female patient was admitted to our clinic with complaints of increasing low back pain, contraction of the abdominal and back muscles, gait disturbance, and anxiety that had started 2 years ago. The patient had been previously admitted to the Physical Medicine and Rehabilitation, Neurology, Rheumatology, and Orthopedic clinics in various health institutions with these complaints. In those clinics, after having been examined with various provisional diagnoses, including ankylosing spondylitis because of low back pain, the patient had experienced various tests. In spinal magnetic resonance imaging, S-shaped minimal scoliosis that had dextrorotatory cervicothoracic and levorotatory lumbar spaces, flattening of the cervical lordosis, and prominence in the lumbar lordosis had been identified. In cranial magnetic resonance, more notably on the right side, an increase in the bilateral hippocampal signal had been observed. Despite all the examinations, no definitive diagnosis was established, and only myorelaxant tablets and symptomatic treatment that comprised physical therapy was prescribed. However, the patient's complaints had not demonstrated regression with those therapies.

The patient who was admitted to our clinic because of increasing severity of her complaints had a history of Hashimoto's thyroiditis and complex partial seizures, and thus, carbamazepine therapy with thyroxine was initiated. During the patient's systemic examination, there was no paresthesia, weakness, urine and fecal incontinence, weight loss, fever, and night sweats. Her physical examination revealed that the waist movements in all directions were severely limited, and there was a significant increase in lumbar lordosis. There was rigidity in the abdominal, paraspinal, hip, and proximal lower extremity muscles. Straight leg raising test was negative. No deficits were detected in the patient's sensory and motor examinations. Deep tendon reflexes were hyperactive in the upper and lower extremities. Plantar response was bilateral flexor, and clonus was not observed. The patient presented with a robotic gait.

In laboratory examinations, biochemistry, including creatine kinase, complete blood count, erythrocyte, sedimentation rate, C-reactive protein, and rheumatoid factor levels were normal. The patient's thyroid function tests, anti-thyroglobulin, and thyroid-stimulating hormone receptor antibody blood values were within normal limits. Her anti-thyroid peroxidase autoantibody score was $89 \mathrm{IU}$ and was positive (normal value: 0-34 IU). Fasting blood glucose and oral glucose tolerance test results were within normal limits.

Because of low back pain, axial and abdominal muscle rigidity, hyperlordosis, and taking the patient's autoimmune disease into account, SPS was considered, and the patient was referred to the neurology department. In EMG, in the lumbar paraspinal and rectus abdominis muscles, continuous motor unit discharge
Table 1. Diagnostic criteria for SPS

Dalakas Criteria:

1. Axial muscle stiffness causing prominent and fixed deformity (lumbar hyperlordosis) in the thoracolumbar paraspinal and abdominal muscles

2. Painful spasms increasing by noise, emotional stress, and tactile stimuli

3. Demonstration of the continuous motor unit activity in the agonist and antagonist muscles through EMG.

4. Absence of other neurological or cognitive impairments that could explain the spasm

5. Positive serology for GAD65 (or amphiphysin) antibodies

6. No response to diazepam

EMG: electromyography; GAD65: glutamic acid decarboxylase65

and simultaneous co-contraction were observed. No evidence of denervation was observed.

Cerebrospinal fluid (CSF) analysis revealed that anti-GAD IGG was positive (anti-GAD $65 ;+++, 1 / 100^{\text {th }}$ of the titer anti GAD67; +++, $1 / 32^{\text {nd }}$ of the titer). No oligoclonal bands were detected in CSF. Serology and viral markers were negative in CSF and serum. With these results SPS diagnosis was confirmed by the neurology department. The patient's tumor markers that were examined for the purpose of paraneoplastic screening and paraneoplastic panel results containing the amphiphiles antibodies were negative. The breast ultrasonography revealed a BI-RADS2 cyst in the right breast and reactive hyperplasia lymph nodes in both the axillary regions. In the electroencephalographic examination, paroxysmal epileptiform disorder in the bilateral temporal, which was more apparent on the left side, was observed. Seizures were reported to be connected to the limbic encephalitis.

The patient was administered IVIG therapy for a period of 5 days. For painful spasms, diazepam and baclofen administrations were initiated. Joint range of motion, stretching, and strengthening exercises for the abdominal muscles were prescribed. On the $3^{\text {rd }}$ and $6^{\text {th }}$ month follow-up visits after the treatment, there was a significant improvement in her gait and low back movements. The scheduled follow-up of the patient was an IVIG therapy once in 3 weeks and a home exercise program. For case presentation, written consent was obtained from the patient.

\section{Discussion}

Stiff person syndrome is a rare autoimmune disease whose exact prevalence is still unknown. It occurs more often among women than men, and it usually occurs when patients are in their 30 s. In approximately $60 \%-80 \%$ of SPS cases, anti-GAD autoantibodies in CSF were found to be positive (1). Moreover, in our case, anti-GAD autoantibodies in CSF were positive.

Glutamic acid decarboxylase is involved in the gamma aminobutyric acid (GABA) synthesis, which is the basic inhibitory transmitter in the brain and spinal cord. Decrease in GAD ac- 
tivity causes a decrease in GABA levels and continuous motor neuronal discharge. Consequently, stiffness and cramps occur in the muscles. However, because different pathways that are responsible for GABA production are present, there are also cases in which GAD autoantibodies are negative (12).

Stiff person syndrome has been associated with autoimmune diseases, such as type $1 \mathrm{DM}$, autoimmune thyroiditis, epilepsy, pernicious anemia, and vitiligo (3). GAD is a major autoantigen in type $1 \mathrm{DM}$. Eighty percent of newly diagnosed type 1 DM cases were positive for anti-GAD (13). In up to $60 \%$ of SPS cases, type $1 \mathrm{DM}$ was identified (7). In our case, the results of fasting blood glucose and oral glucose tolerance tests that had been performed in terms of DM results were within normal limits. However, the patient was being followed in relevant units because of her autoimmune thyroiditis and epilepsy diagnosis.

Some SPS cases paraneoplastically emerge during the course of malignancies, such as breast, ovary, and small-cell lung cancers. In these patients, anti-amphiphiles and -gephyrin antibodies are positive $(3,13)$. In our case, although paraneoplastic panel results containing these autoantibodies were negative, no pathology was detected in malignancy screening tests.

Electromyography is an aid tool used in the diagnosis of SPS. Simultaneous and continuous motor unit activity is observed in the agonist and antagonist muscles. This motor activity is suppressed via diazepam, sleeping, and general anesthesia (7). In our patient, continuous motor unit discharge and simultaneous co-contractions were followed in the lumbar paraspinal and rectus abdominis muscles during the period of rest. These observed motor activities and co-contractions were suppressed with 10 $\mathrm{mg}$ diazepam in accordance with the SPS diagnostic criteria (6).

The symptoms of SPS usually insidiously begin. Rigidity and stiffness in the chest muscles are the earliest symptoms and are caused by the continuous contractions in the lumbar and abdominal muscles. These contractions initially reveal a fluctuating course. As the disease progresses, the rigidity of the lumbar paraspinal muscles, which is characteristic for SPS, causes excessive increase in the lumbar lordosis (hyperlordosis) $(1,14)$. Rigidity spreads from the chest to the hips and proximal lower limb muscles. With time, the gait is affected, and a robotic gait pattern develops. This leads to an increase in the frequency of falling down. Physical limitations and deterioration of posture and gait negatively affect the patients' quality of life (15). In our case, rigidity and stiffness had primarily begun in the lumbar and abdominal muscles and had then spread to the muscles around the hip and to the proximal lower limb muscles. With time, severe movement limitations in her low back and gait disorder developed.

Pain is a common complaint in patients with SPS. Pain, which is localized in the areas in which rigidity is observed, is persistent and worsening of pain might sometimes be the first symptom (1-14). Pain that is localized in the low back is associated with an increased lumbar lordosis and concomitant muscle spasms because of paraspinal muscle rigidity. These spasms are precipitated by audible and tactile stimulation or emotional stress and may be painful enough to cause disability in patients (14). The main complaints of our patient were low back pain and stiffness in the low back and abdominal muscles. These complaints increased in situations of emotional stress, such as exams and loud environments, and thus, our patient's daily life activities and school performance were negatively affected.

In a number of cases, anxiety disorders, depression, and psychiatric disorders such as phobias are observed (1). Anxious behavior was also observed in our case.

In the examination, a tonus increase in the axial and lower extremity muscles; absence of motor and sensory deficits; hyperreflexia in deep tendon reflexes, although Babinski reflex was negative; increase in lumbar lordosis; presence of board-like rigidity in the abdominal and lumbar paraspinal muscles during palpation; cautious gait in slow and wide-based steps; normal cognitive functions; and normal sphincter functions were stated to be monitored (1). These symptoms were also observed during our patient's examination.

The Dalakas criteria are used worldwide to diagnose SPS. Patients who do not meet these criteria are described as atypical SPS (Table 1) (16). As a result of investigations that are conducted with respect to etiology, our patient meets all of the criteria because of the absence of other neurological or cognitive impairment that explains the patient's signs and symptoms, demonstration of continuous motor unit activity in the lumbar paraspinal and rectus abdominis muscles and suppressing this activity via diazepam, anti-GAD autoantibody positivity, and having a response to diazepam treatment.

For medical treatment, there are two basic approaches: GABA-enhancing and immunomodulatory therapy. In GABAenhancing therapy, diazepam is a frequently used benzodiazepine and is very effective in initial treatment. In SPS cases that reveal no response to diazepam, oral and intrathecal baclofen is another treatment option (17). Agents, such as tiagabine and levetiracetam, are also available as an alternative (8). In immunomodulatory therapy, particularly IVIG, plasmapheresis, rituximab (anti-CD20), and corticosteroids are used (17). In our case, similar to the case of Egwuonw et al. (7), after treatment with benzodiazepine, baclofen, and IVIG, regression in symptoms and improvement in examination findings were observed within 1 month. The patient's pain and stiffness decreased and her gait improved, and this situation had a positive impact on her mood and quality of life due to the decrease in limitations in her daily life activities.

\section{Conclusion}

In this case, we described SPS with autoimmune thyroiditis. As a differential diagnosis, SPS, although rarely observed, must be kept in mind in patients, as in our patient, who present to the Physical Medicine and Rehabilitation outpatient clinics with symptoms, such as limitations in low back movements, low back pain, increased lumbar lordosis, paravertebral muscle spasms, gait disorders, because early diagnosis and treatment increase the quality of life, and thus, prevent the development of a long-term disability.

Informed Consent: Written informed consent was obtained from patient who participated in this case.

Peer-review: Externally peer-reviewed. 
Author Contributions: Concept - B.K., Ş.Ö.; Design - B.K., Ş.Ö, E.K.; Supervision - B.K..; Resource - B.K., Ş.Ö., E.K., E.B.; Materials - B.K., Ş.Ö., E.K., E.B.; Data Collection and/or Processing - B.K., Ş.Ö., E.K., E.B.; Analysis and/or Interpretation - B.K., Ş.Ö., E.K., E.B.; Literature Review - B.K., Ş.Ö., E.K., E.B; Writer - B.K., Ş.Ö., E.K., E.B; Critical Review - B.K.

Conflict of Interest: No conflict of interest was declared by the authors.

Financial Disclosure: The authors declared that this study has received no financial support.

\section{References}

1. Hadavi S, Noyce AJ, Leslie RD, Giovannoni G. Stiff person syndrome. Pract Neurol 2011;11:272-82. [CrossRef]

2. Moersch FP, Woltman HW. Progressive fluctuating muscular rigidity and spasm ("stiff-man" syndrome); report of a case and some observations in 13 other cases. Proc Staff Meet Mayo Clin 1956;31:421-7.

3. Dalakas MC. Progress and stiff challenges in understanding the role of GAD-antibodies in stiff-person syndrome. Exp Neurol 2013;247:303-7. [CrossRef]

4. Alexopoulos H, Dalakas MC. A critical update on the immunopathogenesis ofStiff Person Syndrome. Eur J Clin Invest 2010;40:1018-25. [CrossRef]

5. Dalakas MC. Stiff person syndrome: advances in pathogenesis and therapeutic interventions. Curr Treat Options Neurol 2009;11:102-10. [CrossRef]

6. Dalakas MC, Fujii M, Li M, McElroy B. The clinical spectrum of antiGAD antibody-positive patients with stiff-person syndrome. Neurology 2000;:1531-5. [CrossRef]
7. Egwuonwu S, Chedebeau F. Stiff-person syndrome: a case report and review of the literature. J Natl Med Assoc 2010;102:1261-3.

8. Duddy ME, Baker MR. Stiff person syndrome. Front Neurol Neurosci 2009;26:147-65. [CrossRef]

9. De Camilli P, Thomas A, Cofiell R, Folli F, Lichte B, Piccolo G, et al. The synaptic vesicle associated protein amphiphysin is the $128-\mathrm{kD}$ autoantigen of Stiff-Man syndrome with breast cancer. J Exp Med 1993;178:2219-23. [CrossRef]

10. Butler MH, Hayashi A, Ohkoshi N, Villmann C, Becker CM, Feng G, et al. Autoimmunity to gephyrin in Stiff-Man syndrome. Neuron 2000;26:307-12. [CrossRef]

11. Hegyi CA. Physical therapist management of stiff person syndrome in a 24-year-old woman. J Neurol Phys Ther 2011;91:1403-11. [CrossRef]

12. Goodson B, Martin K, Hunt T. Stiff person syndrome presenting with sudden onset of shortness of breath and difficulty moving the right arm: a case report. J Med Case Rep 2010;4:118. [CrossRef]

13. Rakocevic G, Floeter MK. Autoimmune stiff person syndrome and related myelopathies: understanding of electrophysiological and immunological processes. Muscle Nerve 2012;45:623-34. [CrossRef]

14. Gallien P, Durufle A, Petrilli S, Verin M, Brissot R, Robineau S. Atypical lowback pain: stiff-person syndrome. Joint Bone Spine 2002;69:218-21. [CrossRef]

15. Meinck HM, Thompson PD. Stiff man syndrome and related conditions. Mov Disord 2002;17:853-66. [CrossRef]

16. Dalakas MC. Advances in the pathogenesis and treatment of patients with stiff person syndrome. Curr Neurol Neurosci Rep 2008;8:48-55. [CrossRef]

17. Lorish TR, Thorsteinsson G, Howard FM Jr. Stiff-man syndrome updated. Mayo Clin Proc 1989;64:629-36. [CrossRef] 